\title{
Fluoroscopic Sentinel Events in Neuroendovascular Procedures: How to Screen, Prevent, and Address Occurrence
}

\author{
A.Z. Vance, B.D. Weinberg, G.M. Arbique, J.B. Guild, J.A. Anderson, and D.P. Chason
}

\begin{abstract}
SUMMARY: Radiation-induced skin injury during fluoroscopic procedures has been recently addressed by The Joint Commission, which defined prolonged fluoroscopy resulting in a cumulative peak skin dose of $\geq 15$ Gy to a single field as a sentinel event (FSE). Neuroendovascular procedures can be associated with a high radiation skin dose and present risks such as potential FSEs. Managing these risks is the responsibility of the interventional neuroradiologist. In this review, we discuss hospital policies needed for screening and preventing FSEs, methods for minimizing radiation-induced skin injury, and actions necessary to address potential FSEs once they have occurred.
\end{abstract}

ABBREVIATIONS: FSE = fluoroscopic sentinel event; $\mathrm{FT}=$ fluoroscopy time; $\mathrm{K}_{\mathrm{a}, \mathrm{r}}=$ reference air kerma; $\mathrm{P}_{\mathrm{KA}}=$ kerma-area product; $\mathrm{RCA}=$ root cause analysis; $\mathrm{TJC}$ $=$ The Joint Commission

$\mathbf{R}$ adiation-induced skin injury during fluoroscopic procedures has been recently addressed by TJC, which defined a radiation overdose sentinel event from prolonged fluoroscopy (FSE). Compared with other fluoroscopically guided interventions, interventional cardiology and neuroradiology procedures are particularly associated with a high radiation skin dose and thus potential FSEs. ${ }^{1,2}$ Understanding what an FSE is, how it occurs, and what steps can be taken to avoid it is not only essential knowledge but also the responsibility of any physician performing neuroendovascular procedures. ${ }^{2}$

\section{WHAT IS A FLUOROSCOPIC SENTINEL EVENT?}

In 2005, TJC defined the FSE as prolonged fluoroscopy resulting in a cumulative peak skin dose of $\geq 15$ Gy to a single field, which may be accumulated through multiple procedures during a period from 6 months to 1 year. ${ }^{3}$ All accredited hospitals are required to identify and manage FSEs. If an FSE occurs, TJC requires that an RCA be performed within 45 days. The RCA focuses on process improvement rather than assigning blame to individuals and seeks strategies to prevent future events. These improvements must be implemented and monitored.

From the Department of Radiology, University of Texas Southwestern Medical Center, Dallas, Texas.

Please address correspondence to David P. Chason, MD, Department of Radiology, University of Texas Southwestern Medical Center, 5323 Harry Hines Blvd, Dallas, TX 75390; e-mail: david.chason@utsouthwestern.edu

--. Indicates open access to non-subscribers at www.ajnr.org

http://dx.doi.org/10.3174/ajnr.A3185

\section{SCREENING FOR POTENTIAL FLUOROSCOPIC SENTINEL EVENTS}

The cumulative peak skin dose of interest to TJC is not available to the physician at the time of a procedure but only after a detailed investigation by a medical physicist. To identify potential FSEs, the physician and technical staff must monitor dose parameters that the equipment directly reports: $\mathrm{K}_{\mathrm{a}, \mathrm{r}}, \mathrm{P}_{\mathrm{KA}}$, and FT. $\mathrm{K}_{\mathrm{a}, \mathrm{r}}$ is the dose to air at the interventional reference point, typically $15 \mathrm{~cm}$ from the isocenter toward the $\mathrm{x}$-ray tube. ${ }^{4}$ This is the most useful parameter for identifying FSEs, and its display is required by the FDA on all fluoroscopy equipment manufactured after June $2006 .{ }^{5}$ Some machines report $\mathrm{P}_{\mathrm{KA}}$, which reflects the total radiation dose delivered to a patient and may be better suited for estimating a patient's risk of developing cancer. FT is the least accurate proxy for skin dose but may be the only dose parameter available on older systems. Skin dose measurement systems (eg, Caregraph; Siemens, Erlangen, Germany) have met with limited acceptance by the interventional community; however, these may become more desirable as awareness of radiation-induced skin injury grows.

Threshold values used to trigger FSE investigations must be set low enough to catch all real events and high enough to keep physics evaluations within reasonable limits. At our institution, thresholds of $\mathrm{K}_{\mathrm{a}, \mathrm{r}} \geq 6 \mathrm{~Gy}$ and $\mathrm{FT} \geq 150$ minutes are used. On the basis of data from Miller et al, ${ }^{6}$ these values were chosen to isolate all sentinel events while excluding most procedures from investigation. Similar thresholds have been recommended by others as part of a comprehensive patient radiation safety program. ${ }^{7}$ During fluoroscopic procedures, thresholds also provide real-time warnings for the potential of radiation-induced skin injury. 
Clear hospital policies for recording and reporting data are needed. These include: recording dose parameters for all fluoroscopic procedures, specifying who will record data and where, and having a system in place to screen for potential FSEs and to notify the proper personnel. Similar recommendations for recording information required for skin-dose calculations in the patient's medical record have been made by the FDA. ${ }^{8}$ For example, at our institution, dose parameters are recorded by the interventional nurse or technologist and are saved in the patient's electronic medical record. If an investigative threshold is met during a procedure, risk management will be alerted afterward. They will notify the radiation safety officer and the physics department. An automated daily report containing monitored dose parameters of all fluoroscopic procedures performed during the past 6 months on a patient is also sent to the physics department for tracking multiple procedures.

During a procedure, a team approach involving the physician, technologist, and nurse is required to manage the patient's radiation exposure. Adding prior radiation history should become part of the standard "time out" performed. The nurse or technologist can assist by alerting the physician when radiation exposure is nearing investigative thresholds. ${ }^{7}$ The aim is to do what is best for the patient, and the physician must weigh the benefits of the procedure against the potential of skin damage.

\section{MINIMIZING RADIATION-INDUCED SKIN INJURY}

Minimizing the occurrence of radiation-induced skin injury entails educating physicians and staff on FSEs as well as best practices to control skin dose. The interventional neuroradiologist should know the specifics of their particular equipment and be aware of how different settings affect dose. Setup configurations can vary and may change after software upgrades or service.

The physician must be aware of risk factors that could predispose the patient to radiation-induced skin injury. These include smoking, obesity, diabetes mellitus, connective tissue disorders (eg, rheumatoid arthritis, scleroderma), and certain chemotherapy agents. ${ }^{9}$ Disorders resulting from defects in DNA repair genes can also predispose to radiation-induced skin injury. One such disorder of particular concern for the interventional neuroradiologist is ataxia-telangiectasia, in which the patient may need treatment for multiple cerebral or nasopharyngeal vascular malformations. Previously damaged skin from prior irradiation, trauma, surgery, or burns also predisposes to radiation-induced skin injury. 2,9

Technical factors should be optimized for procedure, body part, and patient body habitus before starting the procedure. Low-dose defaults should be in place for frame rate, filter selection, and dose mode (tube voltage/current curve). At our institution, we have achieved substantial reductions in dose by decreasing the fluoroscopic frame rate from 7.5 to 3 frames/second during diagnostic angiography. Judicious use of magnification modes and removal of antiscatter grids in pediatric cases will also reduce dose.

Adjusting study geometry is an easy way of minimizing skin dose. Moving the table to increase x-ray tube-to-patient distance and minimizing the air gap by bringing the image intensifier as close to the patient as possible can substantially reduce skin dose by means of the $1 / \mathrm{r}^{2}$ principle. Using different angulations can help spread skin dose, but severe angles, which can increase tube output, should be avoided. Collimating as much as practical will reduce field overlap from different angles.

\section{FLUOROSCOPIC SENTINEL EVENT INVESTIGATIONS}

Even when good dose management practices are used, complex procedures may lead to high radiation exposure, and the neuroradiologist must take appropriate steps to ensure proper care. First, the patient and primary care physician should be advised of the potential for radiation-induced skin injury and how to detect it. A note should be made in the patient's medical record, and follow-up should be scheduled in the clinic. The patient should be followed for at least 1 year after the procedure, because radiation injuries can have long-term effects. ${ }^{2}$ Any skin reaction seen after a procedure should be assumed to represent radiation injury until another diagnosis is confirmed. The timing and severity of skin reactions depend on dose and the patient risk factors discussed above. ${ }^{9}$ Higher doses result in earlier and more severe effects. Skin injuries can range from transient erythema/epilation ( $>2 \mathrm{~Gy}$ ) to dermal necrosis ( $>15$ Gy) requiring surgical intervention. Transient erythema can occur within hours and typically lasts 24 hours. Epilation and dermal necrosis typically occur at 2-8 weeks and 6-52 weeks, respectively. The timing, frequency, and duration of patient follow-up should be adjusted, depending on dose and patient risk factors.

When radiation exposure exceeds threshold levels, a medical physicist should perform skin-dose estimation. These calculations are complex, with a level of uncertainty depending on how much information is available. It is critical for the team to preserve and record pertinent data. The study should be protected to prevent loss due to storage limitations on the technique device, which is the most complete source of information. Other useful information that the team should record includes patient positioning, gantry geometry, magnification mode, pulse rate, and dose mode. The specifics of the procedure and prior fluoroscopic procedures that may not be readily apparent from the medical record should be discussed with the physicist.

\section{CONCLUSIONS}

Fluoroscopic sentinel events are the responsibility of the interventionalist from the standpoint of both patient safety and accreditation. The interventional neuroradiologist should assume leadership for reducing the risk of such events while maintaining proper patient care. This entails educating members of the interventional team on fluoroscopic sentinel events, using best practices to minimize dose, and ensuring that appropriate hospital policies are in place and followed.

\section{REFERENCES}

1. Miller DL, Balter S, Cole PE, et al. Radiation doses in interventional radiology procedures: the RAD-IR study. Part II. Skin dose. J Vasc Interv Radiol 2003;14:977-90

2. National Council on Radiation Protection and Measurements. Radiation Dose Management for Fluoroscopically-Guided Interventional Medical Procedures. Bethesda, Maryland: National Council on Radiation Protection and Measurements; 2010. NCRP Report No. 168 
3. The Joint Commission. Radiation overdose as a reviewable sentinel event. http://www.jointcommission.org/assets/1/18/Radiation_Overdose.pdf. Accessed January 22, 2012

4. International Electrotechnical Commission. Medical Electrical Equipment-Part 2-43: Particular Requirements for the Safety and Essential Performance of X-Ray Equipment for Interventional Procedures. Geneva, Switzerland: International Electrotechnical Commission; 2000. IEC 60601-2-43 ed 1.0

5. Code of Federal Regulations. Performance standards for ionizing radiation emitting products: Sec 1020.32 Fluoroscopic equipment. Title 21, Volume 8: Part 1020. Available at: http://www.accessdata.fda.gov/ scripts/cdrh/cfdocs/cfcfr/CFRSearch.cfm?FR=1020.32. Accessed January 22,2012

6. Miller DL, Balter S, Cole PE, et al. Radiation doses in interventional radiology procedures: the RAD-IR study. Part I. Overall measures of dose. J Vasc Interv Radiol 2003;14:711-27

7. Steele JR, Jones AK, Ninan EP. Establishing an interventional radiology patient radiation safety program. Radiographics 2012;32: $277-87$

8. US Food and Drug Administration. Avoidance of serious x-ray-induced skin injuries to patients during fluoroscopically-guided procedures. September 9, 1994. http://www.fda.gov/downloads/Radiation EmittingProducts/RadiationEmittingProductsandProcedures/Medi calImaging/MedicalX-rays/ucm116677.pdf. Accessed January 25, 2012

9. Balter S, Hopewell JW, Miller DL, et al. Fluoroscopically guided interventional procedures: a review of radiation effects on patients' skin and hair. Radiology 2010;254:326-41 